\title{
Uncovering the Oral Dysbiotic Microbiota as Masters of Neutrophil Responses in the Pathobiology of Periodontitis
}

\author{
Zsombor Prucsi ${ }^{1}$, Alicja Płonczyńska ${ }^{1}$, Jan Potempa ${ }^{1,2}$ and Maja Sochalska ${ }^{1 *}$ \\ ${ }^{1}$ Department of Microbiology, Faculty of Biochemistry, Biophysics and Biotechnology, Jagiellonian University, Krakow, \\ Poland, ${ }^{2}$ Department of Oral Immunity and Infectious Diseases, University of Louisville School of Dentistry, Louisville, KY, \\ United States
}

\section{OPEN ACCESS}

Edited by:

Carmen Mariana Chifiriuc, University of Bucharest, Romania

Reviewed by:

Geetha Parthasarathy, Tulane University, United States

Eugenia Bezirtzoglou, Democritus University of Thrace,

Greece

Youngnim Choi,

Seoul National University, South Korea

*Correspondence: Maja Sochalska maja.sochalska@uj.edu.pl

Specialty section: This article was submitted to

Microbial Immunology,

a section of the journal

Frontiers in Microbiology

Received: 06 July 2021 Accepted: 14 September 2021 Published: 11 October 2021

Citation:

Prucsi Z, Płonczyńska A, Potempa J and Sochalska M (2021)

Uncovering the Oral Dysbiotic Microbiota as Masters of Neutrophil Responses in the Pathobiology

of Periodontitis.

Front. Microbiol. 12:729717. doi: 10.3389/fmicb.2021.729717
Numerous bacterial species participate in the shift of the oral microbiome from beneficial to dysbiotic. The biggest challenge lying ahead of microbiologists, immunologists and dentists is the fact that the bacterial species act differently, although usually synergistically, on the host immune cells, including neutrophils, and on the surrounding tissues, making the investigation of single factors challenging. As biofilm is a complex community, the members interact with each other, which can be a key issue in future studies designed to develop effective treatments. To understand how a patient gets to the stage of the late-onset (previously termed chronic) periodontitis or develops other, in some cases life-threatening, diseases, it is crucial to identify the microbial composition of the biofilm and the mechanisms behind its pathogenicity. The members of the red complex (Porphyromonas gingivalis, Treponema denticola, and Tannerella forsythia) have long been associated as the cause of periodontitis and stayed in the focus of research. However, novel techniques, such as $16 \mathrm{~S}$ clonal analysis, demonstrated that the oral microbiome diversity is greater than ever expected and it opened a new era in periodontal research. This review aims to summarize the current knowledge concerning bacterial participation beyond $P$. gingivalis and the red complex in periodontal inflammation mediated by neutrophils and to spread awareness about the associated diseases and pathological conditions.

Keywords: periodontitis, neutrophils (PMNs), innate immunity, virulence factor, inflammation

\footnotetext{
Abbreviations: TLR, Toll-like receptor; MMPs, Matrix metalloproteinases; NET, Neutrophil extracellular trap; Msp, major outer sheath protein; eATP, extracellular adenosine triphosphate; NOD-like receptors, nucleotide-binding oligomerization domain-like receptors; HNP-1, Human Neutrophil Peptide-1; hDFSCs, Human dental follicle stem cells; PMNs, polymorphonuclear leukocytes; GSDs, Glycogen storage diseases; S-layer, surface layer; MAPK, mitogen-activated protein kinase; MK2, MAPK-activated protein kinase 2; ROS, reactive oxygen species; IL, interleukin; SCFAs, short-chain fatty acids; Spp., Species; LtxA, leukotoxin A; NE, Neutrophil elastase; CDT, cytolethal distending toxin; MOI, Multiplicity of infection;

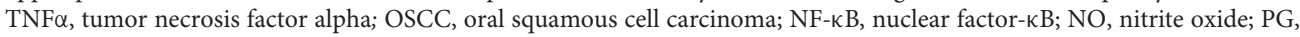
prostaglandin; LPS, lipopolysaccharide; DHMDT, Daehwangmokdantang; ERK, extracellular signal-regulated kinases.
} 


\section{BIOFILM IMPACT ON NEUTROPHILS IN THE DEVELOPMENT OF THE PERIODONTAL DISEASE}

The innate immune system is the first line of defense against pathogenic invasion. The response begins with the recruitment of immune cells. In the oral cavity, the most abundant contributors are neutrophils. The mechanism of the immune system involves the promotion of inflammation, recruitment of other immune cell types and use of neutrophil-specific defense mechanisms (Figure 1). The coordinated attack against pathogens involves the formation of Neutrophil extracellular traps (NETs), a web-like structure destined to capture and eliminate, the internalization (a.k.a. phagocytosis) and the release of the diverse granule content (Scott and Krauss, 2012; Vladimer et al., 2013; Li et al., 2020). In response to the biofilm microbiome, e.g., Fusobacterium nucleatum a significant change in neutrophil gene expression is observed (Wright et al., 2011).

Within the oral biofilm, pathogens have developed countless sophisticated strategies to bypass elimination and turn an inflamed environment in their favor, such as manipulation of neutrophil survival, prolonged inflammatory responses or subversion of anti-microbial properties (White et al., 2014; Olsen and Yilmaz, 2016). Throughout the shift of the microbial composition of the oral cavity, also known as the development of periodontitis, some pathogens act as bridging species between early and late contributors. During the progression of the infection, a strong manipulation of the neutrophil function by the biofilm pathogens can be identified (Table 1). Research-wise, it is highly challenging to reveal the interaction between multispecies biofilms and neutrophils. The first step is to identify pathogen-specific effects.

\section{PATHOGEN-SPECIFIC EFFECTS ON NEUTROPHIL FUNCTIONS}

\section{Fusobacterium nucleatum}

Fusobacterium nucleatum is recognized as a master species in the development of periodontitis, with many strain-specific functions. In order to enhance the multiplication of late colonizers, it is crucial to create favorable conditions with priority to decrease neutrophil efficiency. This is carried out by decreasing superoxide generation and apoptosis induction, limiting the number of counter-attacking immune cells (Kurgan et al., 2017). The production and release of reactive oxygen species (ROS) is part of the pathogen elimination strategies and can be induced by the phagocytosis of the invaders (ElBenna et al., 2016; Zeng et al., 2019). In contrast to wellcharacterized pathogens, the amount of $F$. nucleatum differs within the timeline of disease progression in accordance with its bridging-specie nature (Tomšič et al., 2021). Moreover, it has been proved that $F$. nucleatum exaggerates NET formation in comparison to other bacterial species, such as $P$. gingivalis, in strong connection with NOD-like receptors (Alyami et al., 2019). Triggering NETosis, instead of phagocytosis, as a neutrophil defense mechanism is probably associated with the huge size of the pathogen (Urban et al., 2006). Another antimicrobial mechanism strongly exploited by $F$. nucleatum is the release of Human Neutrophil Peptide-1 (HNP-1) (Musrati et al., 2016). Increased concentrations of HNP-1 peptide trigger epithelial cell death and bacterial attachment to keratinocytes (Gursoy et al., 2013). Exposure of $F$. nucleatum to defensins, a class of antimicrobial peptides released for instance by neutrophils, can result in decreased bacterial membrane permeability and elevated dental plaque biofilm formation. These strategies are destined to aid the resistance against the membrane disruption and lysis caused by the above-mentioned proteins. As a consequence, the proliferation level is secured (Keskin et al., 2014; Xu and Lu, 2020). Importantly, secreted serine protease fusolisin degrades extracellular matrix proteins as well as cleaves the most abundant immunoglobulin at the mucosal surface $(\operatorname{IgA})$, contributing to the inactivation of the host defense and disease progression (Bachrach et al., 2004; Doron et al., 2014). To add insult to injury, observed tissue environment modulation can give rise to other pathological conditions, such as colorectal cancer (Luo et al., 2019).

\section{Prevotella intermedia and Tannerella forsythia}

Pathogens can also have an indirect effect on the function of neutrophils. In the case of Prevotella intermedia and Tannerella forsythia, it has been proved that they can adhere to and internalize into human dental follicle stem cells (hDFSCs). This infection modulates the environment and diminishes the expression of cytokines, however, it does not change hDFSC differentiation capacity. Consequently, a reduced release of IL8 can contribute to limited chemotaxis of polymorphonuclear leukocytes (PMNs). Moreover, in the presence of infected hDFSCs neutrophil phagocytic activity and NET formation are also decreased, which gives pathogens enough time for gingival colonization (Hieke et al., 2016). Importantly, a challenge with $T$. forsythia induces a strong immune response as indicated by the number of immune cells accumulated at the lesion of administration in a murine model. These in vivo experiments showed, that unlike in the presence of $P$. gingivalis, the neutrophil migration is not inhibited (Gosling et al., 2005). However, some comorbidity, such as glycogen storage diseases (GSDs), can in few cases further enhance gingival tissue destruction. A case report of a GSD patient manifesting with the subversion of the neutrophil chemotaxis and neutropenia described an evident $T$. forsythia-mediated intraoral bone loss (Ma et al., 2018).

Interestingly, Ksiazek et al. discovered that $T$. forsythia expresses a serpin (protease inhibitor) called miropin that can contribute to its survival and ability to avoid protease activity of neutrophils (Ksiazek et al., 2015). Strikingly, another newly discovered metalloproteinase called mirolysin together with the previously characterized, secreted metalloproteinase called karilysin, represent important virulence factors of T. forsythia. Both proteinases show a synergistic inhibitory effect on many pathways in the host immune system. Significantly, 

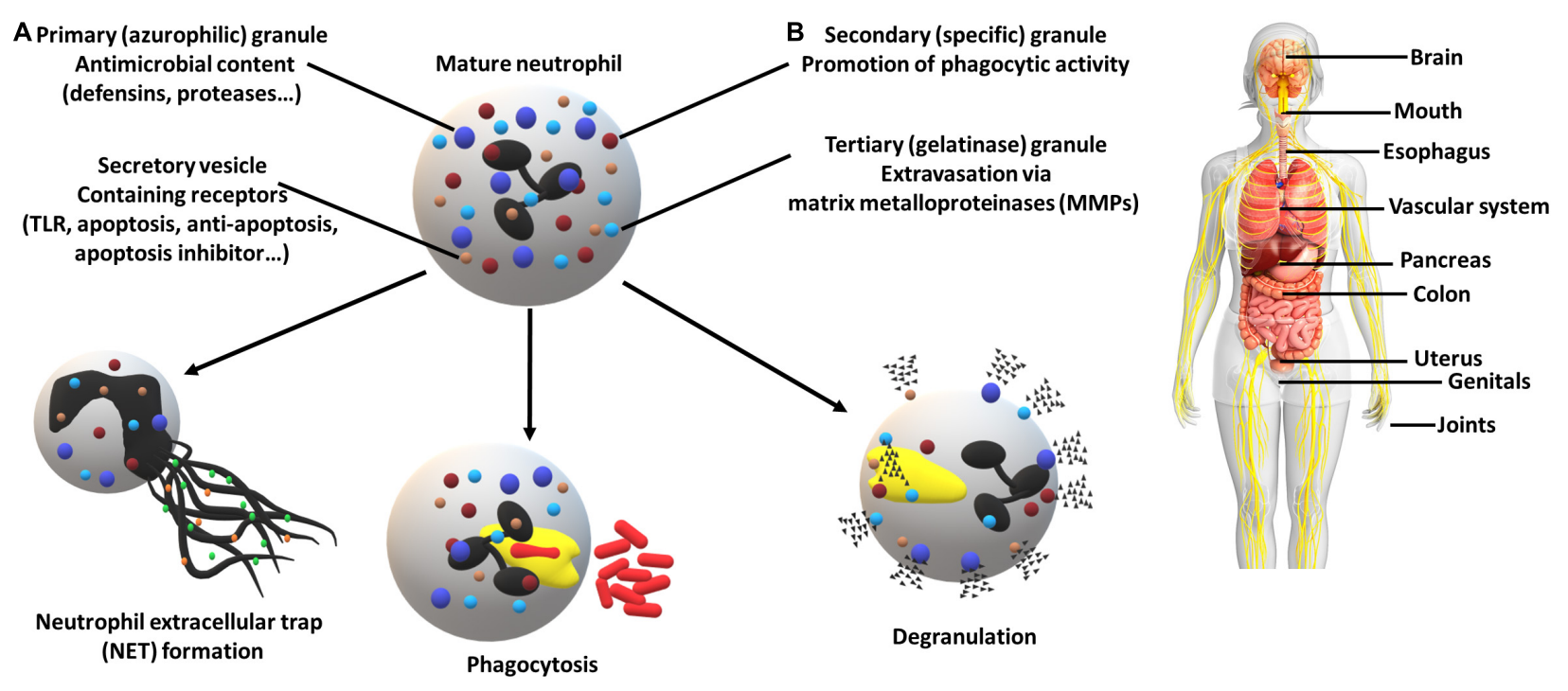

FIGURE 1 | Neutrophil defense mechanisms (A) and associated diseases (B). (A) Neutrophils can employ numerous strategies in order to eliminate pathogens, including the formation of neutrophil extracellular traps, destined to capture and eliminate, internalization (phagocytosis) and the release of a high variety of granule contents (e.g., receptors, proteases and enzymes). The utilization of secretory vesicles provides an easy to mobilize source of receptors crucial for pathogen recognition (TLRs) and cell fate determination (apoptosis regulators). (B) Periodontal pathogens have been associated with multiple other diseases which resulted in increased attention. Affected organs and systems (non-exhaustive): brain (Alzheimer's disease), mouth (periodontitis, oral squamous cell carcinoma, peri-implantitis), esophagus (esophageal cancer), vascular system (aortitis, atherosclerosis), pancreas (pancreatic cancer), colon (colorectal cancer), uterus (preterm birth), genitals (bacterial vaginosis), joints (rheumatoid arthritis).

TABLE 1 | Identified prevalent pathogens associated with periodontitis (non-exhaustive).

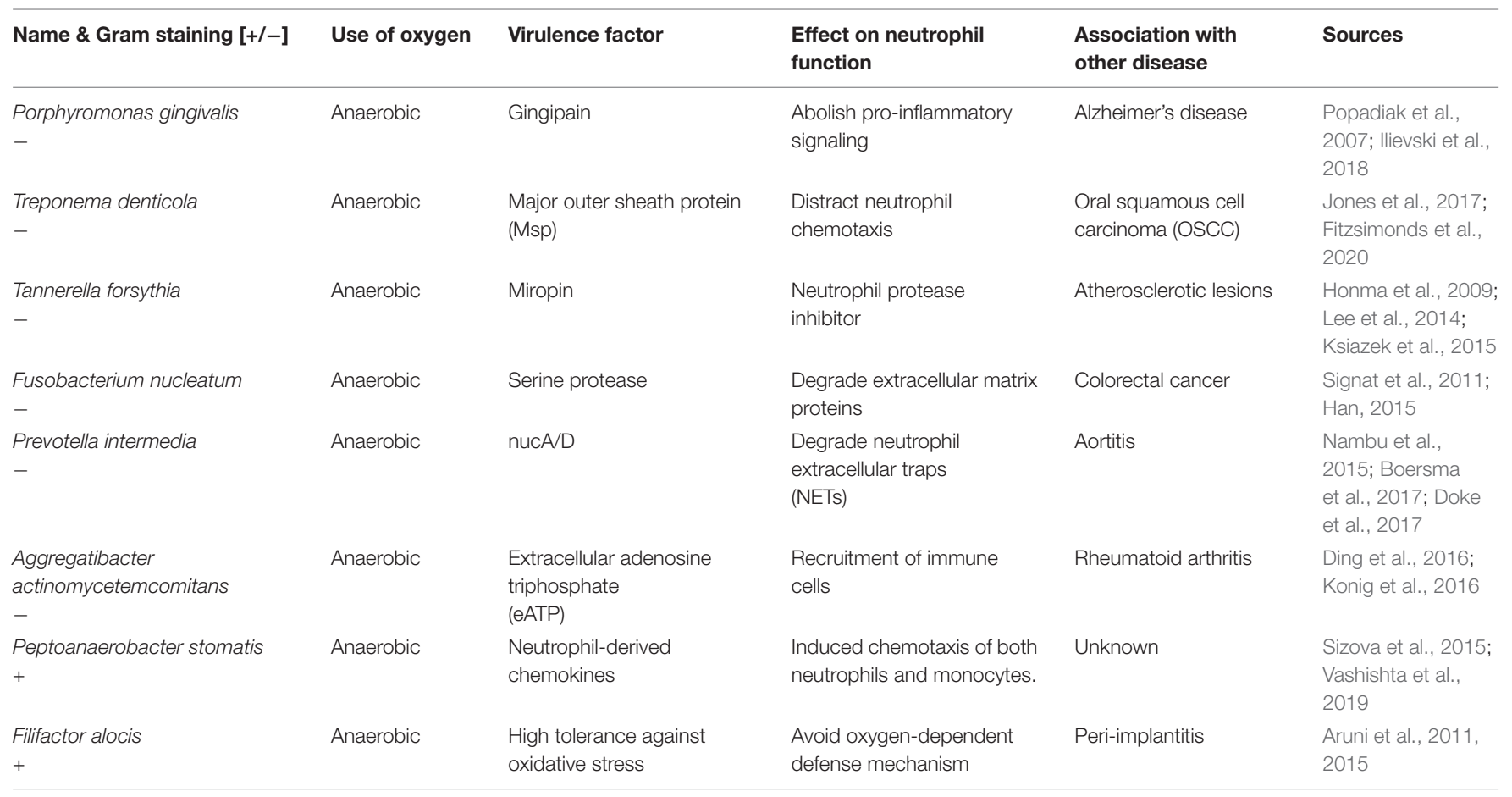

T. forsythia with a mutation in the expression of these enzymes presented with a strongly diminished survival rate (Jusko et al., 2012, 2015). Apart from proteinases, an outer surface layer (S-layer) is a $T$. forsythia-associated virulence factor that can contribute to serum resistance and has a significant role in coaggregation with other oral pathogens, such as $P$. gingivalis. The S-layer significantly reduces the deposition of $\mathrm{C} 3 \mathrm{~b}$ on the bacterial surface, which would 
act as a tag for phagocytosis (Shimotahira et al., 2013). Strikingly, a bacterial glycan found linked to the S-layer can modulate dendritic cells and suppress $\mathrm{T}$ helper 17 response (Settem et al., 2013). As an anaerobic bacteria, T. forsythia lacks a complex enzymatic system against oxidative stress. However, the genome of this pathogen encodes an oxidative stress response sensor protein (OxyR) homolog, that acts as a positive regulator for antioxidant gene expression. This can contribute to the resistance of the bacterial community to oxidative stress in the aerobic oral cavity and protect against oxidative burst in leukocytes, which is essential in the dental plaque biofilm formation (Honma et al., 2009; Moriguchi et al., 2017).

The main component of NETs is DNA (Brinkmann et al., 2004). Among other species (P. gingivalis, F. nucleatum and Aggregatibacter actinomycetemcomitans), $P$. intermedia has the highest nuclease activity, enabling it to block the capture and subsequent phagocytosis by neutrophils. Two genes have been identified as responsible for this feature, nucA and $n u c D$, encoding enzymes that require cations for their activity (Doke et al., 2017). Still, this strategy itself cannot be considered unique, as numerous pathogens associated with periodontal disease are able to express such enzymes, including members of the red and the orange complexes (Palmer et al., 2012). Bacteria species are grouped based on chronological coexistence during diseases progression. Members of the red complex (Porphyromonas gingivalis, Treponema denticola and Tannerella forsythia) are late colonizers and the multiplication of them relies on, and is tightly connected to the conquest of the members of the orange complex (e.g., Fusobacterium nucleatum, Prevotella intermedia) (Mohanty et al., 2019).

\section{Aggregatibacter actinomycetemcomitans}

Secreted extracellular adenosine triphosphate (eATP) is a distinctive virulence factor characterized in A. actinomycetemcomitans. eATP is predominantly an intracellular signaling molecule involved in the recruitment of immune cells (Ding et al., 2016). However, in periodontitis, eATP secreted by A. actinomycetemcomitans induces an upregulation of cytokine expression, resulting in the massive recruitment of inflammatory cells via mainly the p38 mitogenactivated protein kinase (MAPK) and MAPK-activated protein kinase 2 (MK2) pathways (Herbert et al., 2017). Therefore, would be of great importance to investigate the effects of eATP on p38 or MK2 kinases not only in macrophages, but also in neutrophils Interestingly, macrophages challenge with $A$. actinomycetemcomitans induced autophagic influx, restricting the expression of the proinflammatory cytokine IL-1 $\beta$ and ROS production, which ensure protection for this pathogen (Lee et al., 2020). Of importance, bacterial metabolites, such as short-chain fatty acids (SCFAs), are widely spread among different species and connected to a great number of immunological disorders (Ferreira et al., 2014). During bacterial infection, A. actinomycetemcomitans-associated
SCFAs can also alter neutrophil effector mechanisms by downregulating cytokine production and phagocytic activity (Corrêa et al., 2017).

Leukotoxins are virulence factors expressed e.g., by some Staphylococcus spps. or by Mannheimia haemolytica, altering both the innate and the adaptive immune system (Futagawa-Saito et al., 2004; Aulik et al., 2010). Strikingly, leukotoxin A (LtxA) secreted by A. actinomycetemcomitans triggers a dysregulation in neutrophils, resulting in the release of citrullinated proteins (Konig et al., 2016). The hypercitrullination of host proteins, on one hand leads to diminished functions, such as in the case of histone proteins embedded in NETs can be responsible for decreased anti-microbial activity (Li et al., 2010). On the other hand, the development of rheumatoid arthritis (RA) is the consequence of an elevated level of citrullinated proteins, that leads to the hyperactivity of the immune system resulting in the destruction of the host tissue (Kuhn et al., 2006). The link between RA and periodontitis has long been under the scope of scientific research due to the numerous shared immunepathological similarities, including overall disease progression, cytokine profile and risk factors (Koziel et al., 2014; de Molon et al., 2019). As these two diseases can present with similar symptoms, some approaches can be applied in both cases to control disease manifestations, i.e., the melanocortin agonism can be a potential way to overcome excessive oral inflammation (Madeira et al., 2016). Melanocortin proteins upon receptor biding elevate the resolution of inflammation by reducing the amount of released pro-inflammatory cytokines and induce efferocytosis, the clearance of neutrophils by macrophages (Montero-Melendez et al., 2011). Besides the induction of citrullinating enzymes in neutrophils, LtxA has a strong toxic effect on leukocytes and induces NET formation in a dosedependent manner. Additionally, the activity of neutrophil elastase (NE), a principal proteinase in bacterial defense, is exploited. Normally, NE is localized in the cytoplasm, however, upon LtxA exposure, neutrophil lysis is triggered, followed by the release of high amounts of elastase. As a consequence, human gingival epithelial cells and fibroblasts detach and die (Madeira et al., 2016; Hiyoshi et al., 2019).

Of note, A. actinomycetemcomitans activates many more neutrophil defense mechanisms, such as ROS production, the release of proteases and the already mentioned NET formation that can be considered a successful defense strategy in the absence of bacterial nuclease activity (Mikolai et al., 2021). Although, the coin has two sides, the overactivation of the abovementioned mechanisms results in the destruction of the host tissues. Among other virulence factors, this pathogen produces a toxin called the Cytolethal Distending Toxin (CDT), which causes cell cycle arrest in vitro and in vivo as well as blocks proliferation of the target cells. These disease-promoting effects of CTD are noted towards the periodontal epithelial cells in the rat model (Ohara et al., 2011). Unfortunately, amoxicillin, azithromycin, and metronidazole show an attenuated efficiency against A. actinomycetemcomitans, while phagocytosis of the pathogen is only effective at a lower MOI (Multiplicity of Infection) (Ardila and Bedoya-García, 2020). Fortunately, when neutrophils are highly outnumbered by bacterial cells, 
internalized azithromycin significantly increases the phagocytic elimination efficiency of PMNs (Lai et al., 2015).

\section{Peptoanaerobacter stomatis and Filifactor alocis}

Peptoanaerobacter stomatis is a newly characterized member of the destructive oral microbiome. In contrast to well-characterized periodontal pathogens, this one is Gram-positive (Sizova et al., 2015). Infection with this pathogen promotes migration of not just neutrophils, but also monocytes, which additionally strongly fuel inflammation, along with the vigorously induced granule content exocytosis (Vashishta et al., 2019). Furthermore, a significant induction of NET formation is observed upon neutrophil challenge with this pathogen (Armstrong et al., 2018). Moreover, $P$. stomatis is relatively resistant to phagocytosis, while ROS production is significantly increased. These types of defense mechanisms are a double-edged sword, because they simultaneously induce the degradation of the host gingival tissue and periodontitis progression (Flores et al., 2017).

Another Gram-positive member of the community is Filifactor alocis that shows an extraordinary resilience to oxidative stress as mentioned in Table 1. This provides a substantial colonization advantage over the host defense system and competing pathogens. Similar to other victorious pathogens, F. alocis manipulates the neutrophil immune responses. The analysis of global changes in the transcriptome of neutrophils challenged with F. alocis reveals strong effects on the PMNs. A delayed apoptosis is accompanied by a prolonged inflammatory response and activated migration through the MAPK cascade and the TNF- $\alpha$ signaling pathways (Miralda et al., 2020). Strikingly, F. alocis fails to induce NET formation, but doesn't influence the P. stomatis-mediated NETosis. In contrast, an earlier challenge of neutrophils with $F$. alocis decreases NET formation triggered by PMA (Armstrong et al., 2018).

\section{BEYOND PERIODONTITIS}

Recently, periodontal pathogens have started being linked to other, often life-threatening diseases, such as atherosclerosis, cardiovascular diseases or rheumatoid arthritis, as mentioned above (Baetta and Corsini, 2010; Steyers and Miller, 2014). Campylobacter rectus in addition to inhibit neutrophil elastase by ecotin, has been associated with hypertension (Pietropaoli et al., 2019; Thomas et al., 2020). P. gingivalis is the most abundant pathogen of all the detected species (Mougeot et al., 2017). Notwithstanding the fact that inflammation is an essential part of the defense of the immune system, in the case of chronic inflammation, the effect is the opposite. In the oral cavity, a dysbiotic and proinflammatory environment can accelerate the development of gum disease or can even lead to oral cancer. Accordingly, P. gingivalis, F. nucleatum, and Treponema denticola are the most frequently identified enriched species in patients with oral squamous cell carcinoma (OSCC) (Fitzsimonds et al., 2020). As indicated in Table 1, T. denticola can alter neutrophil migration as well as trigger a strong inflammatory response, mediated by the elevated expression of Oncostatin M
(Jones et al., 2020). The identification of $P$. gingivalis and the secreted virulence factor (protease gingipain) in the brains of patients has been an important milestone in the research of Alzheimer's disease. In vivo models demonstrated the pathogen's ability to translocate from the oral cavity to the brain (Ilievski et al., 2018). By applying this knowledge, neurodegeneration can be reduced using specific gingipain inhibitors, which may be a promising treatment (Dominy et al., 2019). Of note, in rare cases, F. nucleatum was isolated from immunocompromised patients with pyogenic liver abscesses. However, this might be a slight contribution based on negligible case numbers (Collins and Diamond, 2021).

\section{SUMMARY AND FUTURE REMARKS}

The scope of this article included anaerobic bacterial species. However, it has to be mentioned that also aerobic species (Streptococcus and Staphylococcus) avoid killing by neutrophils and can be found among other microorganisms leading to periodontal disease (Daniluk et al., 2006). As mentioned and summarized in Table 1, such pathogens start to be linked to other severe disorders and accelerated disease progressions. As a result of emerging detection methods, both the list of pathogens and the linked diseases will grow constantly, providing not just a better understanding, but also powerful diagnostic tools based on biomarkers in the long term (Han et al., 2021). Although it is almost impossible to list all the pathways and virulence factors, Table 1 presents the variety of bacterial adaptation mechanisms. The most abundant virulence factors are the proteases, but they are usually specific for the individual bacterial species. Therefore. such peculiarities hinder the development of new therapeutic approaches.

The shift of the oral microbiome and the emerging inflammation are the results of complex bacterial interactions and biofilm formation. Through the mentioned examples, it is demonstrated that pathogens develop an arsenal of functions to generate a favorable environment and to avoid killing by the most abundant immune cell type found in the oral cavity, the neutrophil. There are still many pathways to be discovered in the future and it cannot be ignored that this is not just a localized problem. Figure 1 illustrates that pathogens can use body fluids as highways to reach other parts of the body and promote inflammation. This might be the key information in some of the adverse pregnancy outcomes, where the placental microbiome shows an incredible resemblance to its assumed origin, the mouth (Fischer et al., 2019).

Interestingly, an investigation of ancient and traditional medications is still as beneficial as at the dawn of modern medicine. Daehwangmokdantang (DHMDT) is a polyherbal mixture known in ancient Korea. Another potential medicament can be an extract from a shrub called border forsythia (in Latin: Forsythia $x$ intermedia) known in ancient China. Active lignans from its leaves or flowers have a similar anti-inflammatory effect through the MAPK/ERK pathway (Michalak et al., 2018). 
Therefore, the review of the underlying molecular mechanisms can bring us closer to efficient treatments (Máthé, 2020). The nuclear factor- $\kappa \mathrm{B}(\mathrm{NF}-\kappa \mathrm{B})$ is the major transcription factor during inflammation that controls the expression of many proinflammatory factors, such as nitrite oxide (NO), prostaglandin (PG)E2, TNF- $\alpha$ and IL-1 $\beta$ (Li and Verma, 2002). Bacterial lipopolysaccharide (LPS) induces the translocation of NF$\kappa \mathrm{B}$ from the cytoplasm to the nucleus. In the presence of DHMDT, the process is inhibited and the expression of the mentioned pro-inflammatory substances is significantly reduced (Lee et al., 2017).

Statins are a class of widely used lipid-lowering medications that also have antimicrobial properties. A novel study by the group of dr Piotr Mydel (Kamińska et al., 2019), aiming to analyze statin effects on a dysbiotic oral microbiome in vitro, included different pathogens, such as $P$. gingivalis, $F$. nucleatum, Actinomyces naeslundii, T. forsythia, and Streptococcus gordonii. Results indicate high effectiveness against $P$. gingivalis without killing the commensal microbiota, which is a side effect of broadspectrum antibiotics.

In summary, the future of periodontal medicine undoubtedly lies in a personalized approach as the microbial composition shows a huge variation between patients. An essential step along the way is the identification of contributing pathogens, their distinct biomarkers and the development of specific diagnostic tools (Van der Weijden et al., 2021).

\section{REFERENCES}

Alyami, H. M., Finoti, L. S., Teixeira, H. S., Aljefri, A., Kinane, D. F., and Benakanakere, M. R. (2019). Role of NOD1/NOD2 receptors in Fusobacterium nucleatum mediated NETosis. Microb. Pathog. 131, 53-64. doi: 10.1016/j. micpath.2019.03.036

Ardila, C. M., and Bedoya-García, J. A. (2020). Antimicrobial resistance of Aggregatibacter actinomycetemcomitans, Porphyromonas gingivalis and Tannerella forsythia in periodontitis patients. J. Glob. Antimicrob. Resist. 22, 215-218. doi: 10.1016/J.JGAR.2020.02.024

Armstrong, C. L., Klaes, C. K., Vashishta, A., Lamont, R. J., and Uriarte, S. M. (2018). Filifactor alocis manipulates human neutrophils affecting their ability to release neutrophil extracellular traps induced by PMA. Innate Immun. 24, 210-220. doi: 10.1177/1753425918767507

Aruni, A. W., Mishra, A., Dou, Y., Chioma, O., Hamilton, B. N., and Fletcher, H. M. (2015). Filifactor alocis-a new emerging periodontal pathogen. Microbes Infect. 17, 517-530. doi: 10.1016/j.micinf.2015.03.011

Aruni, A. W., Roy, F., and Fletcher, H. M. (2011). Filifactor alocis has virulence attributes that can enhance its persistence under oxidative stress conditions and mediate invasion of epithelial cells by porphyromonas gingivalis. Infect. Immun. 79, 3872-3886. doi: 10.1128/IAI.05631-11

Aulik, N. A., Hellenbrand, K. M., Klos, H., and Czuprynski, C. J. (2010). Mannheimia haemolytica and its leukotoxin cause neutrophil extracellular trap formation by bovine neutrophils. Infect. Immun. 78, 4454-4466. doi: 10.1128/ IAI.00840-10

Bachrach, G., Haake, S. K., Glick, A., Hazan, R., Naor, R., Andersen, R. N., et al. (2004). Characterization of the novel Fusobacterium nucleatum plasmid pKH9 and evidence of an addiction system. Appl. Environ. Microbiol. 70, 6957-6962. doi: 10.1128/AEM.70.12.6957-6962.2004

Baetta, R., and Corsini, A. (2010). Role of polymorphonuclear neutrophils in atherosclerosis: current state and future perspectives. Atherosclerosis 210, 1-13. doi: 10.1016/J.ATHEROSCLEROSIS.2009.1 0.028

Boersma, C., Kampschreur, L. M., Buter, H., Doorenbos, B. M., Klinkert, P., and Koning, G. G. (2017). Prevotella intermedia infection causing acute and

\section{AUTHOR CONTRIBUTIONS}

ZP wrote and revised the manuscript, prepared figures, and tables. AP wrote and revised the manuscript. MS wrote, corrected, and revised the manuscript. JP corrected the manuscript. All authors contributed to the article and approved the submitted version.

\section{FUNDING}

This work was supported by funding grants from the Foundation for Polish Science (First Team/2017-4/40, POIR.04.04.00-0042FE/17) to MS, US NIH/NIDCR (DE026280) to JP and International Visegrad Fund (\#51910968) to ZP.

\section{ACKNOWLEDGMENTS}

The authors acknowledge the financial support from the Foundation for Polish Science grant FIRST TEAM. The FIRST TEAM program is co-financed by the European Union under the European Regional Development Fund. The open-access publication of this article was funded by the Priority Research Area BioS as part of the program "Excellence Initiative - Research University" at the Jagiellonian University in Krakow, Poland.

complicated aortitis-a case report. Int. J. Surg. Case Rep. 32, 58-61. doi: 10.1016/ j.ijscr.2017.02.015

Brinkmann, V., Reichard, U., Goosmann, C., Fauler, B., Uhlemann, Y., Weiss, D. S., et al. (2004). Neutrophil extracellular traps kill bacteria. Science 303, 1532-1535. doi: 10.1126/SCIENCE.1092385

Collins, L., and Diamond, T. (2021). Fusobacterium nucleatum causing a pyogenic liver abscess: a rare complication of periodontal disease that occurred during the COVID-19 pandemic. BMJ Case Reports 14, e240080. doi: 10.1136/bcr-2020240080

Corrêa, R. O., Vieira, A., Sernaglia, E. M., Lancellotti, M., Vieira, A. T., AvilaCampos, M. J., et al. (2017). Bacterial short-chain fatty acid metabolites modulate the inflammatory response against infectious bacteria. Cell. Microbiol. 19:e12720. doi: 10.1111/CMI.12720

Daniluk, T., Tokajuk, G., Cylwik-Rokicka, D., Rozkiewicz, D., Zaremba, M. L., and Stokowska, W. (2006). Aerobic and anaerobic bacteria in subgingival and supragingival plaques of adult patients with periodontal disease. Adv. Med. Sci. 51(Suppl. 1), 81-85.

de Molon, R. S., Rossa, C. Jr., Thurlings, R. M., Cirelli, J. A., and Koenders, M. I. (2019). Linkage of periodontitis and rheumatoid arthritis: current evidence and potential biological interactions. Int. J. Mol. Sci. 20, 4541. doi: 10.3390/ ijms20184541

Ding, Q., Quah, S. Y., and Tan, K. S. (2016). Secreted adenosine triphosphate from Aggregatibacter actinomycetemcomitans triggers chemokine response. Mol. Oral Microbiol. 31, 423-434. doi: 10.1111/omi.12143

Doke, M., Fukamachi, H., Morisaki, H., Arimoto, T., Kataoka, H., and Kuwata, H. (2017). Nucleases from Prevotella intermedia can degrade neutrophil extracellular traps. Mol. Oral Microbiol. 32, 288-300. doi: 10.1111/omi.12171

Dominy, S. S., Lynch, C., Ermini, F., Benedyk, M., Marczyk, A., Konradi, A., et al. (2019). Porphyromonas gingivalis in Alzheimer's disease brains: evidence for disease causation and treatment with small-molecule inhibitors. Sci. Adv. 5:eaau3333. doi: 10.1126/sciadv.aau3333

Doron, L., Coppenhagen-Glazer, S., Ibrahim, Y., Eini, A., Naor, R., Rosen, G., et al. (2014). Identification and characterization of fusolisin, the Fusobacterium nucleatum autotransporter serine protease. PLoS One 9:e111329. doi: 10.1371/ journal.pone.0111329 
El-Benna, J., Hurtado-Nedelec, M., Marzaioli, V., Marie, J.-C., Gougerot-Pocidalo, M.-A., and Dang, P. M.-C. (2016). Priming of the neutrophil respiratory burst: role in host defense and inflammation. Immunol. Rev. 273, 180-193. doi: 10. 1111/imr.12447

Ferreira, C. M., Vieira, A. T., Vinolo, M. A. R., Oliveira, F. A., Curi, R., and Martins, F. D. S. (2014). The central role of the gut microbiota in chronic inflammatory diseases. J. Immunol. Res. 2014, 1-12. doi: 10.1155/2014/689492

Fischer, L. A., Demerath, E., Bittner-Eddy, P., and Costalonga, M. (2019). Placental colonization with periodontal pathogens: the potential missing link. Am. J. Obstet. Gynecol. 221, 383-392.e3. doi: 10.1016/j.ajog.2019.04.029

Fitzsimonds, Z. R., Rodriguez-Hernandez, C. J., Bagaitkar, J., and Lamont, R. J. (2020). From beyond the pale to the pale riders: the emerging association of bacteria with oral cancer. J. Dental Res. 99, 604-612. doi: 10.1177/ 0022034520907341

Flores, E. J., Tian, S., Sizova, M., Epstein, S. S., Lamont, R. J., and Uriarte, S. M. (2017). Peptoanaerobacter stomatis primes human neutrophils and induces granule exocytosis. Infect. Immun. 85:e01043-16. doi: 10.1128/IAI.01043- 16

Futagawa-Saito, K., Sugiyama, T., Karube, S., Sakurai, N., Ba-Thein, W., and Fukuyasu, T. (2004). Prevalence and characterization of leukotoxin-producing Staphylococcus intermedius in isolates from dogs and pigeons. J. Clin. Microbiol. 42, 5324-5326. doi: 10.1128/JCM.42.11.5324-5326.2004

Gosling, P. T., Gemmell, E., Carter, C. L., Bird, P. S., and Seymour, G. J. (2005). Immunohistological analysis of Tannerella forsythia-induced lesions in a murine model. Oral Microbiol. Immunol. 20, 25-30. doi: 10.1111/j.1399-302X. 2004.00188.x

Gursoy, U. K., Könönen, E., Luukkonen, N., and Uitto, V.-J. (2013). Human neutrophil defensins and their effect on epithelial cells. J. Periodontol. 84, 126-133. doi: 10.1902/jop.2012.120017

Han, P., Bartold, P. M., Salomon, C., and Ivanovski, S. (2021). Salivary outer membrane vesicles and dna methylation of small extracellular vesicles as biomarkers for periodontal status: a pilot study. Int. J. Mol. Sci. 22, 1-15. doi: $10.3390 /$ ijms 22052423

Han, Y. W. (2015). Fusobacterium nucleatum: a commensal-turned pathogen. Curr. Opin. Microbiol. 23, 141-147. doi: 10.1016/j.mib.2014.11.013

Herbert, B. A., Steinkamp, H. M., Gaestel, M., and Kirkwood, K. L. (2017). Mitogen-activated protein kinase 2 signaling shapes macrophage plasticity in Aggregatibacter actinomycetemcomitans-induced bone loss. Infect. Immun. 85:e00552-16. doi: 10.1128/IAI.00552-16

Hieke, C., Kriebel, K., Engelmann, R., Müller-Hilke, B., Lang, H., and Kreikemeyer, B. (2016). Human dental stem cells suppress PMN activity after infection with the periodontopathogens Prevotella intermedia and Tannerella forsythia. Sci. Rep. 6:39096. doi: 10.1038/srep39096

Hiyoshi, T., Domon, H., Maekawa, T., Nagai, K., Tamura, H., Takahashi, N., et al. (2019). Aggregatibacter actinomycetemcomitans induces detachment and death of human gingival epithelial cells and fibroblasts via elastase release following leukotoxin-dependent neutrophil lysis. Microbiol. Immunol. 63, 100-110. doi: 10.1111/1348-0421.12672

Honma, K., Mishima, E., Inagaki, S., and Sharma, A. (2009). The OxyR homologue in Tannerella forsythia regulates expression of oxidative stress responses and biofilm formation. Microbiology 155, 1912-1922. doi: 10.1099/mic.0.027920-0

Ilievski, V., Zuchowska, P. K., Green, S. J., Toth, P. T., Ragozzino, M. E., Le, K., et al. (2018). Chronic oral application of a periodontal pathogen results in brain inflammation, neurodegeneration and amyloid beta production in wild type mice. PLoS One 13:e0204941. doi: 10.1371/journal.pone.0204941

Jones, M. M., Vanyo, S. T., Ibraheem, W., Maddi, A., and Visser, M. B. (2020). Treponema denticola stimulates Oncostatin $\mathrm{M}$ cytokine release and de novo synthesis in neutrophils and macrophages. J. Leukocyte Biol. 108, 1527-1541. doi: 10.1002/JLB.4MA0620-072RR

Jones, M. M., Vanyo, S. T., and Visser, M. B. (2017). The C-terminal region of the major outer sheath protein of Treponema denticola inhibits neutrophil chemotaxis. Mol. Oral Microbiol. 32, 375-389. doi: 10.1111/omi.12180

Jusko, M., Potempa, J., Karim, A. Y., Ksiazek, M., Riesbeck, K., Garred, P., et al. (2012). A metalloproteinase karilysin present in the majority of Tannerella forsythia isolates inhibits all pathways of the complement system. J. Immunol. 188, 2338-2349. doi: 10.4049/jimmunol.1101240

Jusko, M., Potempa, J., Mizgalska, D., Bielecka, E., Ksiazek, M., Riesbeck, K., et al. (2015). A metalloproteinase mirolysin of Tannerella forsythia inhibits all pathways of the complement system. J. Immunol. 195, 2231-2240. doi: 10.4049/ jimmunol.1402892
Kamińska, M., Aliko, A., Hellvard, A., Bielecka, E., Binder, V., Marczyk, A., et al. (2019). Effects of statins on multispecies oral biofilm identify simvastatin as a drug candidate targeting Porphyromonas gingivalis. J. Periodontol. 90, 637-646. doi: 10.1002/JPER.18-0179

Keskin, M., Könönen, E., Söderling, E., Isik, G., Firatli, E., Uitto, V.-J., et al. (2014). Increased proliferation and decreased membrane permeability as defense mechanisms of Fusobacterium nucleatum against human neutrophilic peptide1. Anaerobe 30, 35-40. doi: 10.1016/j.anaerobe.2014.08.001

Konig, M. F., Abusleme, L., Reinholdt, J., Palmer, R. J., Teles, R. P., Sampson, K., et al. (2016). Aggregatibacter actinomycetemcomitans-induced hypercitrullination links periodontal infection to autoimmunity in rheumatoid arthritis. Sci. Transl. Med. 8:369ra176. doi: 10.1126/scitranslmed.aaj1921

Koziel, J., Mydel, P., and Potempa, J. (2014). The link between periodontal disease and rheumatoid arthritis: an updated review. Curr. Rheumatol. Rep. 16:408. doi: 10.1007/s11926-014-0408-9

Ksiazek, M., Mizgalska, D., Enghild, J. J., Scavenius, C., Thogersen, I. B., and Potempa, J. (2015). Miropin, a novel bacterial serpin from the periodontopathogen Tannerella forsythia, inhibits a broad range of proteases by using different peptide bonds within the reactive center loop. J. Biol. Chem. 290, 658-670. doi: 10.1074/jbc.M114.601716

Kuhn, K. A., Kulik, L., Tomooka, B., Braschler, K. J., Arend, W. P., Robinson, W. H., et al. (2006). Antibodies against citrullinated proteins enhance tissue injury in experimental autoimmune arthritis. J. Clin. Invest. 116, 961-973. doi: 10.1172/JCI25422

Kurgan, Ş, Kansal, S., Nguyen, D., Stephens, D., Koroneos, Y., Hasturk, H. et al. (2017). Strain-specific impact of Fusobacterium nucleatum on neutrophil function. J. Periodontol. 88, 380-389. doi: 10.1902/jop.2016.160212

Lai, P.-C., Schibler, M. R., and Walters, J. D. (2015). Azithromycin enhances phagocytic killing of Aggregatibacter actinomycetemcomitans Y4 by human neutrophils. J. Periodontol. 86, 155-161. doi: 10.1902/jop.2014.140183

Lee, H. A., Park, M. H., Song, Y., Na, H. S., and Chung, J. (2020). Role of Aggregatibacter actinomycetemcomitans - induced autophagy in inflammatory response. J. Periodontol. 91, 1682-1693. doi: 10.1002/JPER.19-0639

Lee, H. R., Jun, H. K., and Choi, B. K. (2014). Tannerella forsythia BspA increases the risk factors for atherosclerosis in $\mathrm{ApoE}^{-/-}$mice. Oral Dis. 20, 803-808. doi: 10.1111/odi.12214

Lee, M., Hong, S., Park, C., Han, M., Kim, S., Hong, S., et al. (2017) Anti-inflammatory effects of Daehwangmokdantang, a traditional herbal formulation, in lipopolysaccharide-stimulated RAW 264.7 macrophages. Exp. Ther. Med. 14, 5809-5816. doi: 10.3892/etm.2017.5296

Li, P., Li, M., Lindberg, M. R., Kennett, M. J., Xiong, N., and Wang, Y. (2010). PAD4 is essential for antibacterial innate immunity mediated by neutrophil extracellular traps. J. Exp. Med. 207, 1853-1862. doi: 10.1084/jem.20100239

Li, Q., and Verma, I. M. (2002). NF-кB regulation in the immune system. Nat. Rev. Immunol. 2, 725-734. doi: 10.1038/nri910

Li, T., Zhang, Z., Li, X., Dong, G., Zhang, M., Xu, Z., et al. (2020). Neutrophil extracellular traps: signaling properties and disease relevance. Mediators Inflamm. 2020, 1-14. doi: 10.1155/2020/9254087

Luo, K., Zhang, Y., Xv, C., Ji, J., Lou, G., Guo, X., et al. (2019). Fusobacterium nucleatum, the communication with colorectal cancer. Biomed. Pharmacother. 116:108988. doi: 10.1016/j.biopha.2019.108988

Ma, R., Moein Vaziri, F., Sabino, G., Sarmast, N., Zove, S., Iacono, V., et al. (2018). Glycogen storage disease $\mathrm{Ib}$ and severe periodontal destruction: a case report. Dent. J. 6:53. doi: 10.3390/dj6040053

Madeira, M. F. M., Queiroz-Junior, C. M., Montero-Melendez, T., Werneck, S. M. C., Corrêa, J. D., Soriani, F. M., et al. (2016). Melanocortin agonism as a viable strategy to control alveolar bone loss induced by oral infection. FASEB J. 30, 4033-4041. doi: 10.1096/fj.201600790R

Máthé, Á (2020). Medicinal and Aromatic Plants of North America, Vol. 6. Cham: Springer, 1-342. doi: 10.1007/978-3-030-44930-8

Michalak, B., Filipek, A., Chomicki, P., Pyza, M., Woźniak, M., Żyżyńska-Granica, B., et al. (2018). Lignans from forsythia $\mathrm{x}$ intermedia leaves and flowers attenuate the pro-inflammatory function of leukocytes and their interaction with endothelial cells. Front. Pharmacol. 9:401. doi: 10.3389/fphar.2018.00401

Mikolai, C., Branitzki-Heinemann, K., Ingendoh-Tsakmakidis, A., Stiesch, M., von Köckritz-Blickwede, M., and Winkel, A. (2021). Neutrophils exhibit an individual response to different oral bacterial biofilms. J. Oral Microbiol. 13:1856565. doi: $10.1080 / 20002297.2020 .1856565$ 
Miralda, I., Vashishta, A., Rogers, M. N., Rouchka, E. C., Li, X., Waigel, S., et al. (2020). Whole transcriptome analysis reveals that filifactor alocis modulates TNF $\alpha$-stimulated MAPK activation in human neutrophils. Front. Immunol. 11:497. doi: 10.3389/fimmu.2020.00497

Mohanty, R., Asopa, S., Joseph, M. D., Singh, B., Rajguru, J., Saidath, K., et al. (2019). Red complex: polymicrobial conglomerate in oral flora: a review. J. Fam. Med. Prim. Care 8, 3480. doi: 10.4103/jfmpc.jfmpc_759_19

Montero-Melendez, T., Patel, H. B., Seed, M., Nielsen, S., Jonassen, T. E. N., and Perretti, M. (2011). The melanocortin agonist AP214 exerts anti-inflammatory and proresolving properties. Am. J. Pathol. 179, 259-269. doi: 10.1016/J. AJPATH.2011.03.042

Moriguchi, K., Hasegawa, Y., Higuchi, N., Murakami, Y., Yoshimura, F., Nakata, K., et al. (2017). Energy dispersive spectroscopy-scanning transmission electron microscope observations of free radical production in human polymorphonuclear leukocytes phagocytosing non-opsonized Tannerella forsythia. Microsc. Res. Tech. 80, 555-562. doi: 10.1002/jemt.22819

Mougeot, J.-L. C., Stevens, C. B., Paster, B. J., Brennan, M. T., Lockhart, P. B., and Mougeot, F. K. B. (2017). Porphyromonas gingivalis is the most abundant species detected in coronary and femoral arteries. J. Oral Microbiol. 9:1281562. doi: 10.1080/20002297.2017.1281562

Musrati, A. A., Fteita, D., Paranko, J., Könönen, E., and Gürsoy, U. K. (2016). Morphological and functional adaptations of Fusobacterium nucleatum exposed to human neutrophil Peptide-1. Anaerobe 39, 31-38. doi: 10.1016/j. anaerobe.2016.02.008

Nambu, T., Yamane, K., Maruyama, H., Mashimo, C., and Yamanaka, T. (2015). Complete genome sequence of Prevotella intermedia strain 17-2. Genome Announc. 3:e00951-15. doi: 10.1128/genomeA.00951-15

Ohara, M., Miyauchi, M., Tsuruda, K., Takata, T., and Sugai, M. (2011). Topical application of Aggregatibacter actinomycetemcomitans cytolethal distending toxin induces cell cycle arrest in the rat gingival epithelium in vivo. J. Periodontal Res. 46, 389-395. doi: 10.1111/j.1600-0765.2011. 01348.x

Olsen, I., and Yilmaz, Ö (2016). Modulation of inflammasome activity by Porphyromonas gingivalis in periodontitis and associated systemic diseases. J. Oral Microbiol. 8:30385. doi: 10.3402/jom.v8.30385

Palmer, L. J., Chapple, I. L. C., Wright, H. J., Roberts, A., and Cooper, P. R. (2012). Extracellular deoxyribonuclease production by periodontal bacteria. J. Periodontal Res. 47, 439-445. doi: 10.1111/j.1600-0765.2011.01451.x

Pietropaoli, D., Del Pinto, R., Ferri, C., Ortu, E., and Monaco, A. (2019). Definition of hypertension-associated oral pathogens in NHANES. J. Periodontol. 90, 866-876. doi: 10.1002/JPER.19-0046

Popadiak, K., Potempa, J., Riesbeck, K., and Blom, A. M. (2007). Biphasic effect of gingipains from Porphyromonas gingivalis on the human complement system. J. Immunol. 178, 7242-7250. doi: 10.4049/jimmunol.178.11.7242

Scott, D. A., and Krauss, J. (2012). Neutrophils in periodontal inflammation. Front. Oral Biol. 15:56-83. doi: 10.1159/000329672

Settem, R. P., Honma, K., Nakajima, T., Phansopa, C., Roy, S., Stafford, G. P., et al. (2013). A bacterial glycan core linked to surface (S)-layer proteins modulates host immunity through Th17 suppression. Mucosal Immunol. 6, 415-426. doi: 10.1038/mi.2012.85

Shimotahira, N., Oogai, Y., Kawada-Matsuo, M., Yamada, S., Fukutsuji, K., Nagano, K., et al. (2013). The surface layer of Tannerella forsythia contributes to serum resistance and oral bacterial coaggregation. Infect. Immun. 81, 1198-1206. doi: 10.1128/IAI.00983-12

Signat, B., Roques, C., Poulet, P., and Duffaut, D. (2011). Fusobacterium nucleatum in periodontal health and disease. Curr. Issues Mol. Biol. 13, 25-36. doi: 10. 21775/cimb.013.025
Sizova, M. V., Chilaka, A., Earl, A. M., Doerfert, S. N., Muller, P. A., Torralba, M., et al. (2015). High-quality draft genome sequences of five anaerobic oral bacteria and description of Peptoanaerobacter stomatis gen. nov., sp. nov., a new member of the family Peptostreptococcaceae. Stand. Genomic Sci. 10:37. doi: 10.1186/s40793-015-0027-8

Steyers, C., and Miller, F. (2014). Endothelial dysfunction in chronic inflammatory diseases. Int. J. Mol. Sci. 15, 11324-11349. doi: 10.3390/ijms150711324

Thomas, C., Nothaft, H., Yadav, R., Fodor, C., Alemka, A., Oni, O., et al. (2020). Characterization of ecotin homologs from Campylobacter rectus and Campylobacter showae. PLoS One 15:e0244031. doi: 10.1371/journal.pone. 0244031

Tomšič, K., Rodič, K., Sotošek, A., Videmšek, P., Seme, K., Herrera, D., et al. (2021). Do differences in cultivable subgingival species exist between different periodontitis stages and grades? Oral Health Prev. Dentistry 19, 15-24. doi: 10.3290/j.ohpd.b875525

Urban, C. F., Reichard, U., Brinkmann, V., and Zychlinsky, A. (2006). Neutrophil extracellular traps capture and kill Candida albicans yeast and hyphal forms. Cell. Microbiol. 8, 668-676. doi: 10.1111/j.1462-5822.2005.00659.x

Van der Weijden, F., Rijnen, M., and Valkenburg, C. (2021). Comparison of three qPCR-based commercial tests for detection of periodontal pathogens. Sci. Rep. 11:6141. doi: 10.1038/s41598-021-85305-3

Vashishta, A., Jimenez-Flores, E., Klaes, C., Tian, S., Miralda, I., Lamont, R., et al. (2019). Putative periodontal pathogens, Filifactor alocis and Peptoanaerobacter stomatis, induce differential cytokine and chemokine production by human neutrophils. Pathogens 8:59. doi: 10.3390/pathogens8020059

Vladimer, G. I., Marty-Roix, R., Ghosh, S., Weng, D., and Lien, E. (2013). Inflammasomes and host defenses against bacterial infections. Curr. Opin. Microbiol. 16, 23-31. doi: 10.1016/j.mib.2012.11.008

White, P., Cooper, P., Milward, M., and Chapple, I. (2014). Differential activation of neutrophil extracellular traps by specific periodontal bacteria. Free Radic. Biol. Med. 75(Suppl. 1):S53. doi: 10.1016/j.freeradbiomed.2014.10.827

Wright, H. J., Chapple, I. L. C., Matthews, J. B., and Cooper, P. R. (2011). Fusobacterium nucleatum regulation of neutrophil transcription. J. Periodontal Res. 46, 1-12. doi: 10.1111/j.1600-0765.2010.01299.x

$\mathrm{Xu}, \mathrm{D}$., and Lu, W. (2020). Defensins: a double-edged sword in host immunity. Front. Immunol. 11:764. doi: 10.3389/fimmu.2020.00764

Zeng, M. Y., Miralda, I., Armstrong, C. L., Uriarte, S. M., and Bagaitkar, J. (2019). The roles of NADPH oxidase in modulating neutrophil effector responses. Mol. Oral Microbiol. 34, 27-38. doi: 10.1111/omi. 12252

Conflict of Interest: The authors declare that the research was conducted in the absence of any commercial or financial relationships that could be construed as a potential conflict of interest.

Publisher's Note: All claims expressed in this article are solely those of the authors and do not necessarily represent those of their affiliated organizations, or those of the publisher, the editors and the reviewers. Any product that may be evaluated in this article, or claim that may be made by its manufacturer, is not guaranteed or endorsed by the publisher.

Copyright (c) 2021 Prucsi, Płonczyńska, Potempa and Sochalska. This is an openaccess article distributed under the terms of the Creative Commons Attribution License (CC BY). The use, distribution or reproduction in other forums is permitted, provided the original author(s) and the copyright owner(s) are credited and that the original publication in this journal is cited, in accordance with accepted academic practice. No use, distribution or reproduction is permitted which does not comply with these terms. 